\title{
Effects of commercial pectinases application on selected properties of red pitaya juice
}

\begin{abstract}
Pitaya or dragon fruits are becoming more popular in Malaysia due to their nutritional benefits. These exotic fruits can be utilized to produce many functional products such as fruit juice. This work examined the effects of commercial enzymes, i.e., Pectinex Ultra SP-L and Pectinex CLEAR on the chemical composition, vitamin $\mathrm{C}$ and total polyphenols contents of red pitaya (Hylocereus polyrhizus) juice, which is the most popular variety of pitaya. We have also observed the differences of these properties in fresh fruit pulp and juice produced without enzymatic treatment. The pitaya juice produced after enzymation and pasteurization has not shown marked changes in the most of its major chemical parameters such as moisture, ash, fat, carbohydrate and calorie. However, enzymation using Pectinex CLEAR leads to the juice with higher yield of protein. The protein content after the treatment increased to $2.20 \% \mathrm{w} / \mathrm{w}$ from $0.23 \% \mathrm{w} / \mathrm{w}$ of the raw juice. Vitamin $\mathrm{C}$ content in treated samples had reduced slightly because of the heat treatment. However, phenolics amounts were slightly higher up to $7 \%$ in the treated samples which suggest that pitaya beverage is rich in antioxidant capacity than in the unprocessed fruit. This study demonstrated the effects of pectinases application on several properties of red pitaya juice with an attempt to obtain some interesting and valuable data for the fruit processing industries. The data obtained were helpful for the optimization of red pitaya juice production as a step toward promoting the potential of red pitaya juice as a functional beverage.
\end{abstract}

Keyword: Pectinases; Pitaya; Fruit processing 